\title{
Factorial analysis optimization of a Diltiazem kinetic spectrophotometric quantification method
}

\author{
João M.M. Leitão ${ }^{a}$, Joaquim C.G. Esteves da Silva ${ }^{b, *}$ \\ a Laboratório de Métodos Instrumentais de Análise, Faculdade de Farmácia da Universidade de Coimbra, Centro de Estudos \\ Farmacêuticos, 3000-432 Coimbra, Portugal \\ b Centro de Investigação em Química, Departamento de Química, Faculdade de Ciências da Universidade do \\ Porto, R. Campo Alegre 687, 4169-007 Porto, Portugal
}

\section{A R T I C L E I N F O}

Article history:

Received 8 July 2007

Received in revised form

14 December 2007

Accepted 18 December 2007

Published on line 27 December 2007

Keywords:

Antihypertensor Diltiazem

UV-Vis spectroscopy

Kinetic method

Optimization

Experimental design methodologies

\begin{abstract}
A B S T R A C T
A Diltiazem kinetic spectrophotometric method was optimized by factorial analysis. The experimental method is based on a two-stage reaction of Diltiazem with hydroxylamine and a ferric salt: in the first stage there is a hydroxamic acid formation; and, in the second stage there is a red colour complex ferric hydroxamate formation. The variables under investigation were: solvent; hydroxylamine, sodium hydroxide and ammonium ferric sulphate concentrations; volume of perchloric acid; and, temperature. The responses of the reactional system were the maximum absorbance, the wavelength and the reaction time at maximum absorbance. Experimental design methodologies were used in the optimization. Fractional and full factorial designs followed by optimization Box-Behnken and central composite experimental designs were used. The observed optimum conditions were: methanol as reaction solvent; hydroxylamine concentration of $9.375 \%$; sodium hydroxide concentration of $18.750 \%$; ferric reagent concentration of $2.000 \%$; minimum volume of perchloric acid to neutralize the sodium hydroxide; and, room temperature as reaction temperature. With this set of experimental conditions a reaction time of $10.5 \mathrm{~s}$ with maximum colour development at $512 \mathrm{~nm}$ wavelength was achieved.
\end{abstract}

(c) 2007 Elsevier B.V. All rights reserved.

\section{Introduction}

The chemical reaction of esters and anhydrides with hydroxylamine $\left(\mathrm{NH}_{2} \mathrm{OH}\right)$ and a ferric salt originates a coloured compound and this reaction is used for their identification [1]. This reaction was also used for the spectrophotometric quantification of esters, anhydrides and organic acids after derivatization [2]. In this reaction, schematically shown in Fig. 1, the esters and anhydrides of carboxylic acids initially react with hydroxylamine in a basic medium, forming an alcohol and a hydroxamic acid (RCONHOH). In a second step, a ferric hydroxamate chelate will be obtained after the neutralization of the sodium hydroxide by the reaction of hydroxamic acid with ferric ions. The lactones that could be considered cyclic esters also react in a similar way [2].

Attending to the analytical potential of this reaction the influence of different experimental variables, the stability of the coloured complex formed and the reaction mechanism were studied by several authors [2-4]. The variables investigated were the solvent, hydroxylamine concentration, amount in excess of the added acid, reaction temperature and reaction time. For many esters the reaction does not take place at room temperature after $30 \mathrm{~min}$ and only at higher temperatures the colour development and the stability of the complex were considered satisfactory. The formation and stability of the complex formed are also affected by the ferric ion concen-

\footnotetext{
* Corresponding author. Tel.: +351 220402569; fax: +351 220402659.

E-mail address: jcsilva@fc.up.pt (J.C.G. Esteves da Silva).
} 


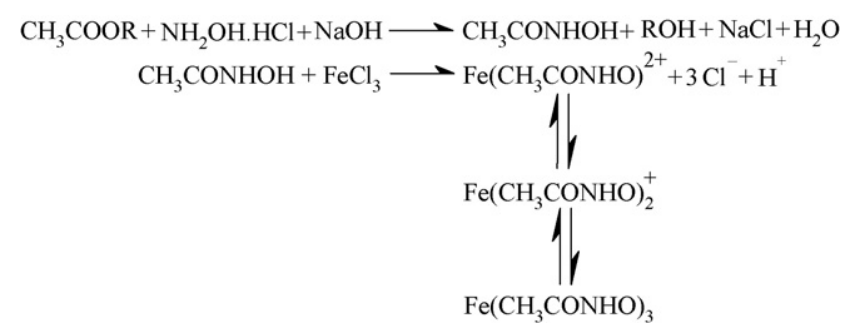

Fig. 1 - Chemical reaction of an ester of the acetic acid with hydroxylamine, intermediary formation of an hydroxamic acid and posterior complex formation by reaction with $\mathrm{Fe}^{3+}$ ions.

tration and by the excess of acid needed for the neutralization of the initially used sodium hydroxide. The ferric ion concentration is considered important for the colour development and the stability of the complex. In Ref. [2] a concentration of $4.8 \mathrm{mM}$ and in Ref. [4] a concentration of $30 \mathrm{mM}$ is indicated as the minimum ideal ferric ion concentrations. Low concentrations of the acid were found more favourable to the complex formation even if it is referred that at lower concentrations of acid a lowest stability of the complex is found.

Diltiazem, one of the major cardiovascular drugs, is a calcium channel blocker widely used as an anti-anginal and anti-hypertensive agent. This drug is officially in the United States, European and British Pharmacopoeia [5-7]. Chemically, Diltiazem hydrochloride belongs to the benzothiazepine class of compounds. The absorption spectrum of Diltiazem in different solvents is characterized by a maximum at approximately 209-240 nm [5-8].

Diltiazem has an ester group and the above-described reaction can be used for its identification and quantification. Two previous studies report the spectrophotometric quantification of Diltiazem with the reaction of this compound with hydroxylamine and ferric ions $[9,10]$. In both studies the hydroxylamine reagent used is prepared by mixing equal volumes of hydroxylamine and sodium hydroxide $12.5 \%\left(\mathrm{~m} \mathrm{~V}^{-1}\right)$, the ferric salt used is dissolved in perchloric acid diluted and the reaction temperature is approximately $70^{\circ} \mathrm{C}$. Besides these experimental conditions used for Diltiazem quantification ethanol was used as solvent of the standard solution and methanol as solvent of the hydroxylamine reagent, the ferric salt is the ferric perchlorate and the reaction time is $5 \mathrm{~min}$ [9]. Also, methanol was used as solvent for the standard solution, water as solvent of the hydroxylamine reagent, the ferric salt is the ammonium ferric sulphate and the reaction time is $20 \mathrm{~min}$ [10]. However these methods suffer interferences and a previous chromatographic separation [9] or chloroform extraction [10] was proposed. The coloured derivative shows a maximum absorbance at wavelength about $500 \mathrm{~nm}$ with linear work range from about 50 to several hundred $\mathrm{mg} / \mathrm{L}$. Other colour development reactions were used for Diltiazem quantification [11-18]. Due to interferences these spectrophotometric methods are usually based on derivative reactions or coupled to extraction techniques to increase selectivity [13-18].
The reaction of Diltiazem with hydroxylamine, leading to the formation of a red coloured complex of ferric hydroxamate, allows the determination of Diltiazem at higher wavelengths. In order to a rapid, sensible and robust quantification of Diltiazem with minimum interferences, the reaction should be optimized. In this paper, the optimal conditions of this reaction, i.e. a minimum reaction time and a maximum absorbance at a highest wavelength (maximum sensitivity with minimum interferences), were obtained using experimental design methodologies [19-23].

A kinetic spectrophtometric analytical methodology based on a second-order multivariate calibration was developed with this optimized reaction. Good results were found with this method in the quantification of Diltiazem in bulk drug and in different pharmaceutical formulations. The results obtained by the UV-Vis direct and UV-Vis second-order multivariate calibration methodologies were compared with the United States Pharmacopoeia HPLC-UV method [24]

\section{Experimental}

\subsection{Reagents}

Diltiazem (99\% purity) reference standard, ammonium ferric sulphate and hydroxylamine hydrochloride were obtained from Sigma-Aldrich Química S.A. (Spain). Sodium hydroxide, methanol for liquid chromatography, isopropanol and ethanol pro-analysis were purchased from Merck, Darmstadt (Germany). Deionised water with resistivity higher than $4 \mathrm{M} \Omega \mathrm{cm}^{-1}$ was used.

\subsection{Solutions}

Diltiazem was dried during $2 \mathrm{~h}$ in an oven at $105^{\circ} \mathrm{C}$. A standard stock solution was prepared in methanol, ethanol, isopropanol and water by rigorous weighting to a final concentration of $8.870 \times 10^{-3} \mathrm{M}$. The hydroxylamine hydrochloride, sodium hydroxide and ammonium ferric sulphate solutions are also prepared by rigorous weighting. Aqueous solutions of hydroxylamine hydrochloride and sodium hydroxide and perchloric acid $7 \%(w / w)$ solutions of ammonium ferric sulphate are prepared. The hydroxylamine reagent used for the colour development reaction was obtained by mixing equal volumes of the aqueous hydroxylamine hydrochloride and sodium hydroxide solutions in the required concentrations of each of the compounds.

In the experimental design optimization the dilutions of all the reagents and of the Diltiazem standard stock solution to a concentration of $5.544 \times 10^{-4} \mathrm{M}$ was obtained with the solvent used to a final volume of $2.5 \mathrm{~mL}$ in the $1 \mathrm{~cm}$ quartz cells. For the initial evaluations the dilutions of all the reagents and of the Diltiazem standard stock solution were performed to a final volume of $25 \mathrm{~mL}$. A standard solution of Diltiazem $1.064 \times 10^{-3} \mathrm{M}$ was obtained. The diluted standard solutions were obtained by rigorous dilution of the initial standard stock solution. Except for the perchloric acid addition the different dilutions of the reagents in the different concentrations used were obtained by adding a fixed volume of the initial solutions. 


\subsection{Instrumentation}

Absorbance measurements were made in a Hewlett-Packard HP8452A diode-array spectrophotometer in advanced mode. In a way to evaluate the reaction time the spectra were collected with the shutter open in $1 \mathrm{~cm}$ quartz cells in a wavelength range from 240 to $800 \mathrm{~nm}$ with a 2-nm interval, run time $60 \mathrm{~s}$, cycle time $1 \mathrm{~s}$ and an integration time $0.5 \mathrm{~s}$. Heating was achieved by the circulation of water from a thermostated water bath.

\subsection{Analytical methodology}

The reaction of Diltiazem with hydroxylamine and ferric ions were performed in a 1-cm quartz cells. The mixture of the reagents is done by stirring with a micro-magnetic bar. The order of reagents mixing is: (i) suitable volume of Diltiazem standard solution in order to obtain a final concentration of $5.544 \times 10^{-4} \mathrm{M}$; (ii) suitable volume of solvent to $0.500 \mathrm{~mL}$ (attending to the previous added volume); (iii) $0.300 \mathrm{~mL}$ of hydroxylamine reagent; (iv) suitable volume of the solvent used to $2.5 \mathrm{~mL}$ (attending to the all the volumes added); (v) variable volume of $7 \%$ perchloric acid (vi) $0.100 \mathrm{~mL}$ of ammonium ferric sulphate. The addition of ammonium ferric sulphate is made after the initiate acquisition of the spectra. The initial evaluation of the reaction of Diltiazem with hydroxylamine and ferric ions was performed in $25.00-\mathrm{mL}$ volumetric flasks.

\subsection{Experimental design optimization}

Attending to the characteristics of this two-step reaction, each of the reaction steps at different $\mathrm{pH}$, it was found necessary to perform this optimization using two reaction types in order to evaluate the excess of perchloric acid solution added. In the first, reaction type A, a fixed concentration of sodium hydroxide $12.5 \%$ was used and the volume of perchloric acid solution added was changed. In the second, reaction type $\mathrm{B}, \mathrm{a}$ minimum volume of the perchloric acid solution was used to neutralize the sodium hydroxide previously added at different concentrations. The first set of experimental design variables evaluated were the solvent, hydroxylamine, sodium hydroxide and ammonium ferric sulphate concentrations, volume of perchloric acid $7 \%$ added and temperature of reaction. The reference values of the factors evaluated were established attending to a previous preliminary univariate analysis. The response variables monitored from the experimental data [time $(\mathrm{s}) \times$ wavelength $(\mathrm{nm})$ ] were the maximum absorbance, wavelength and reaction time at maximum absorbance. The sequence of experiences for all the experimental designs was preformed randomly.

The general strategy was based on a preliminary evaluation of all the factors and the possible interactions between factors by screening using fractional and full factorials experimental designs $[25,26]$, followed by an optimization of the significant factors using a Box-Behnken and Central Composite Designs [26,27]. After a first optimization by a Box-Behnken Design of all the significant factors a Central Composite Design still allows the optimization of some significant factors with additional levels.
The significance of the main effects and the variable interactions were evaluated using higher orders interactions as comparison. When center samples are used a curvature checking of the response is done. These two tests are presented respectively as HOIE and "center" tests [26]. Also, for the initial confirmatory screening designs an evaluation of the critical effects was done by two different statistical methods at significance level of 0.05: (i) a test in which the calculation of the critical effects is based on an estimation of error by the two-factor interactions effects [28]; and (ii) a test in which the calculation of the critical effects based on the algorithm of Dong [29]. The evaluation of the global linear model, global quadratic model, quadratic effects and shape of the response surface is done by analysis of variance (ANOVA) through the F-ratio, respective $p$ value and by the value of multiple correlation coefficients of the response variables $[19,21-23,26]$. Beside this evaluation the minimum, maximum or saddle point estimated of the response surfaces are also presented.

The experimental design procedures are implemented by Unscrambler ${ }^{\circledR}$ program version 7.51 from Camo ASA, Norway.

\section{Results and discussion}

\subsection{Preliminary analysis}

The initial evaluations of the reaction of Diltiazem with hydroxylamine and ferric ions were performed in $25-\mathrm{mL}$ volumetric flasks with a $1.064 \times 10^{-3} \mathrm{M}$ Diltiazem standard solution in methanol. The results obtained confirmed the expected wavelength of maximum absorbance $(500 \mathrm{~nm})$ and the necessity of adding enough volume of acid to neutralize the sodium hydroxide in order to avoid the ferric hydroxide precipitation. Besides that, there is a rapid colour development at room temperature without heating.

In this previous evaluation the different factors that could affect the reaction were evaluated in a univariate way using half and double of the concentrations (sodium hydroxide, hydroxylamine and ferric reagent) usually indicated in the literature, a reaction temperature from room temperature to $70^{\circ} \mathrm{C}$ and the solvents ethanol, methanol, isopropanol and water. Under these conditions it was found that: (i) the minimum sodium hydroxide concentration needed for the reaction colour development is $8 \%$; (ii) an increase of hydroxylamine concentration demands an increase of sodium hydroxide concentration for the reaction colour development; (iii) sodium hydroxide concentration increase causes an absorbance increase; (iv) ferric reagent concentration increase causes an absorbance increase; (v) the same absorbance is found with concentration of hydroxylamine $6.250 \%$ and $12.500 \%$; (vi) higher absorbances are observed with the solvents methanol and ethanol, but methanol is associated with a lower reaction time; (vii) the increase of the reaction temperature did not affect significantly the reaction time and the absorbance.

Attending to this preliminary evaluation, where an absorbance around 1 was observed with a $1.064 \times 10^{-3} \mathrm{M}$ Diltiazem standard, a $5.544 \times 10^{-4} \mathrm{M}$ Diltiazem standard solution with an absorbance around 0.5 was used in the subse- 
Table 1 - Levels of design variables used and range of results of the three responses variables obtained with the

exploratory screening experimental designs for both reaction types in a evaluation of Diltiazem standard $5.544 \times 10^{-4} \mathrm{M}$ with a sodium hydroxide concentration of $\mathbf{1 2 . 5 0 0} \%$ for reaction type $A$ and with a fixed volume of perchloric acid for reaction type B

Design variables

Reaction type

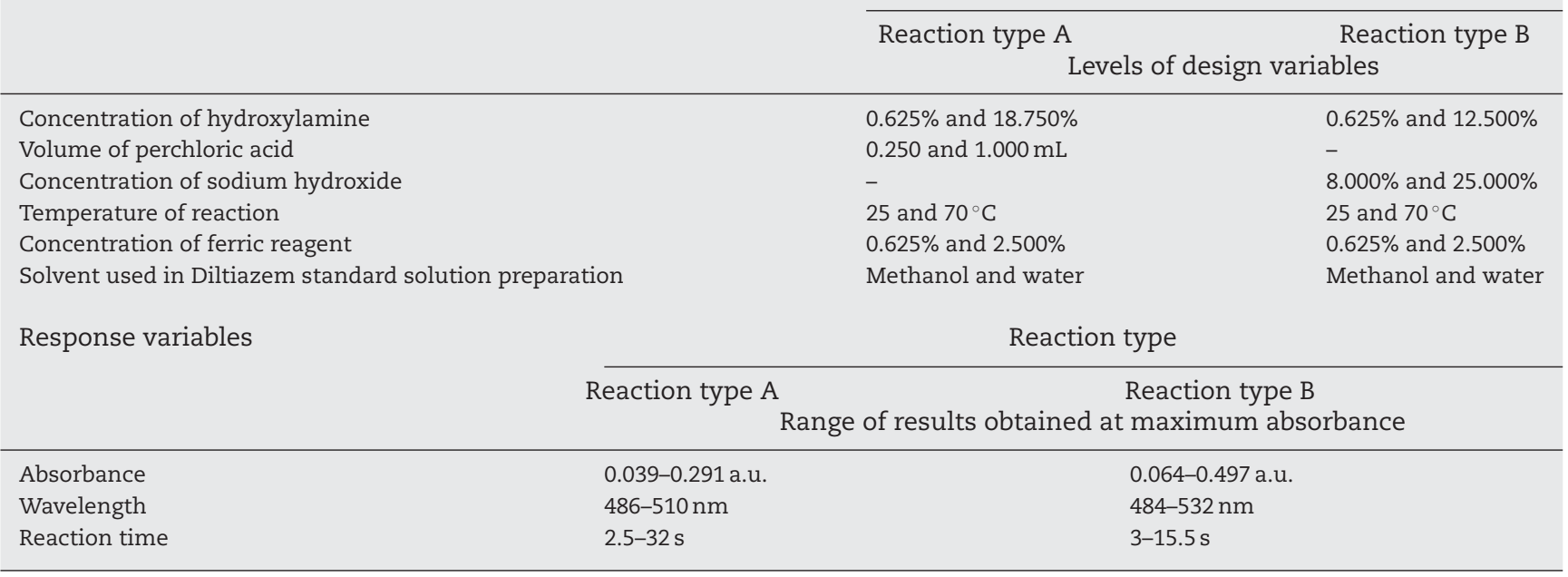

quent study. The values of the experimental design variables were $12.500 \%$ for hydroxylamine and sodium hydroxide concentrations, $1.250 \%$ for ferric reagent concentration, room temperature and methanol as solvent.

\subsection{Screening experimental designs}

\subsubsection{Exploratory screening experimental designs}

Attending to the fact that two different reaction designs are being studied, initial screening $2^{5-2}$ fractional factorial designs were performed with eight experiments each-five design variables, four continuous and one category, each one with two levels and three response variables. With this design the main effects are confounded with the two variable interactions. The levels of the variables for the hydroxylamine, sodium hydrox- ide concentrations and ferric reagent concentrations were established considering as average values the reference concentrations found in the preliminary analysis. For the reaction temperature, and attending to the already lower reaction time found at room temperature, the room temperature was chosen as the minimum level. For the volume of perchloric acid a great range in excess was choose for reaction type A. Also for the solvent, and attending to the preferential use of water as solvent, this solvent was still evaluated. Table 1 presents the design variables studied, the levels of each one and the range of results at the maximum absorbance obtained for the response variables.

From the global analysis of the results obtained for the two reaction types, shown in Table 1, it is important to refer that higher absorbance values and lower reaction time are

Table 2 - Levels of design variables used and range of results of the three response variables obtained with the

confirmatory screening experimental designs for both reaction types in an evaluation of Diltiazem standard

$5.544 \times 10^{-4} \mathrm{M}$, in methanol with a sodium hydroxide concentration of $12.500 \%$ for reaction type $A$ and with a fixed volume of perchloric acid for reaction type $B$

Design variables

Reaction type

Reaction type A

Levels of design variables

Reaction type B

Concentration of hydroxylamine

Volume of perchloric acid

Concentration of sodium hydroxide

Temperature of reaction

Concentration of ferric reagent

Response variables
$6.250 \%$ and $18.750 \%$, center sample, $12.500 \%$ $0.250 \mathrm{~mL}$ and $1.000 \mathrm{~mL}$, center sample, $0.625 \mathrm{~mL}$

25 and $70^{\circ} \mathrm{C}$, center sample, $47.5^{\circ} \mathrm{C}$

$0.625 \%$ and $1.875 \%$, center sample, $-1.250 \%$
$0.625 \%$ and $12.500 \%$, center sample, $-6.563 \%$

$8.000 \%$ and $18.750 \%$, center sample, $-13.375 \%$

25 and $70^{\circ} \mathrm{C}$, center sample, $47.5^{\circ} \mathrm{C}$

$0.625 \%$ and $1.875 \%$, center sample, $-1.250 \%$

\begin{tabular}{lll} 
& \multicolumn{2}{c}{ Reaction type } \\
\cline { 2 - 3 } & \multicolumn{1}{c}{$\begin{array}{c}\text { Reaction type B } \\
\text { Reaction type A } \\
\text { Range of results obtained at maximum absorbance }\end{array}$} \\
\hline Absorbance & $0.105-0.533$ a.u. & $0.074-0.455$ a.u. \\
Wavelength & $486-512 \mathrm{~nm}$ & $484-510 \mathrm{~nm}$ \\
Reaction time & $2-22.5 \mathrm{~s}$ & $2.5-7 \mathrm{~s}$ \\
\hline
\end{tabular}


Table 3 - Results obtained with the confirmatory screening of experimental design for reaction type $A$ by analysis of the main effects and interactions through the

response evaluation of a Diltiazem standard $5.544 \times 10^{-4} \mathrm{M}$, in methanol and with a sodium hydroxide concentration of $12.500 \%{ }^{\mathrm{a}}$

Design variables

Response variables

\begin{tabular}{|c|c|c|c|c|c|c|c|c|c|}
\hline & \multicolumn{3}{|c|}{ Absorbance } & \multicolumn{3}{|c|}{ Wavelength } & \multicolumn{3}{|c|}{ Reaction time } \\
\hline & HOIE & Center & Coefficient $b$ & HOIE & Center & Coefficient $b$ & HOIE & Center & Coefficient $b$ \\
\hline Hydroxylamine (A) & $176.132(0.000)$ & $995.338(0.001)$ & -0.058 & $2.253(0.172)$ & $31.688(0.030)$ & +1.625 & $0.465(0.515)$ & $0.484(0.559)$ & +0.750 \\
\hline Perchloric acid (B) & $765.444(0.000)$ & $4326.000(0.000)$ & -0.122 & $3.853(0.085)$ & $54.188(0.018)$ & +2.125 & $0.545(0.481)$ & $0.568(0.530)$ & +0.813 \\
\hline Temperature (C) & $34.140(0.000)$ & $192.927(0.005)$ & -0.026 & $3.000(0.122)$ & $42.188(0.023)$ & -1.875 & $6.532(0.034)$ & $6.811(0.121)$ & -2.813 \\
\hline Ferric reagent (D) & $154.005(0.000)$ & $870.297(0.001)$ & +0.055 & $7.053(0.029)$ & $99.188(0.010)$ & -2.875 & $1.045(0.337)$ & $1.090(0.406)$ & -1.125 \\
\hline$A B=C D$ & $12.539(0.008)$ & 70.859 (0.014) & +0.016 & $1.613(0.240)$ & $22.688(0.041)$ & -1.375 & $1.290(0.289)$ & $1.345(0.366)$ & +1.250 \\
\hline$A C=B D$ & $22.378(0.002)$ & $126.458(0.008)$ & +0.021 & $5.880(0.042)$ & $82.688(0.012)$ & +2.625 & $0.826(0.390)$ & $0.861(0.451)$ & -1.000 \\
\hline $\mathrm{AD}=\mathrm{BC}$ & $8.007(0.022)$ & $45.246(0.041)$ & +0.013 & $2.253(0.172)$ & $31.688(0.030)$ & +1.625 & $0.390(0.550)$ & $0.407(0.589)$ & -0.688 \\
\hline \multicolumn{10}{|c|}{ Global linear model (ANOVA) } \\
\hline F-ratio $(p)$ & $167.523(0.000)$ & $3.701(0.043)$ & $1.585(0.266)$ & & & & & & \\
\hline Multiple correlation & 0.997 & 0.874 & 0.762 & & & & & & \\
\hline
\end{tabular}

a In HOIE and "center" tests the F-ratio is presented for each main effect and interaction; coefficient $b$ : $b$ regression coefficient of each effect or interaction in the model; in brackets is presented the probability value $(p)$, for a $5 \%$ significance level, the number in brackets indicates in the main or interactions effect evaluation the probability of a main or interaction effects be significant by HOIE or "center" tests or in the global linear model evaluation the probability of the model be significantly valid.

Table 4 - Results obtained with the confirmatory screening experimental design for reaction type B by analysis of the main effects and interactions through the response evaluation of a Diltiazem standard $5.544 \times 10^{-4} \mathrm{M}$, in methanol and with a fixed volume of perchloric acid ${ }^{\mathrm{a}}$

\begin{tabular}{|c|c|c|c|c|c|c|c|c|c|}
\hline \multirow[t]{3}{*}{ Design variables } & \multicolumn{9}{|c|}{ Response variables } \\
\hline & \multicolumn{3}{|c|}{ Absorbance } & \multicolumn{3}{|c|}{ Wavelength } & \multicolumn{3}{|c|}{ Reaction time } \\
\hline & HOIE & Center & Coefficient $b$ & HOIE & Center & Coefficient $b$ & HOIE & Center & Coefficient $b$ \\
\hline Hydroxylamine (A) & $321.353(0.000)$ & $96.429(0.010)$ & +0.102 & $256.889(0.000)$ & $96.333(0.010)$ & +8.500 & $0.010(0.922)$ & $0.188(0.707)$ & +0.031 \\
\hline Sodium hydroxide (B) & $85.586(0.000)$ & $25.682(0.037)$ & +0.053 & $18.000(0.003)$ & $6.750(0.122)$ & -2.250 & $0.258(0.625)$ & $4.688(0.163)$ & +0.156 \\
\hline Temperature (C) & $13.315(0.007)$ & $3.995(0.184)$ & -0.021 & $8.000(0.022)$ & $3.000(0.225)$ & -1.500 & $0.505(0.497)$ & $9.188(0.094)$ & -0.219 \\
\hline Ferric reagent (D) & $21.812(0.002)$ & $6.545(0.125)$ & +0.027 & $80.222(0.000)$ & $30.083(0.032)$ & -4.750 & $0.093(0.768)$ & $1.688(0.324)$ & -0.094 \\
\hline $\mathrm{AB}=\mathrm{CD}$ & $71.398(0.000)$ & $21.425(0.044)$ & +0.048 & $43.556(0.000)$ & $16.333(0.056)$ & +3.500 & $0.258(0.625)$ & $4.688(0.163)$ & +0.156 \\
\hline $\mathrm{AC}=\mathrm{BD}$ & $0.001(0.978)$ & $0.000(0.989)$ & -0.0002 & $80.222(0.000)$ & $30.083(0.032)$ & +4.750 & $1.247(0.297)$ & $22.688(0.041)$ & -0.344 \\
\hline $\mathrm{AD}=\mathrm{BC}$ & $0.158(0.701)$ & $0.048(0.848)$ & -0.002 & $8.000(0.022)$ & $3.000(0.225)$ & +1.500 & $2.320(0.166)$ & $42.188(0.023)$ & -0.469 \\
\hline \multicolumn{10}{|c|}{ Global linear model (ANOVA) } \\
\hline F-ratio $(p)$ & $73.375(0.000)$ & $70.698(0.000)$ & $0.670(0.695)$ & & & & & & \\
\hline Multiple correlation & 0.992 & 0.992 & 0.608 & & & & & & \\
\hline
\end{tabular}


Table 5 - Levels of design variables used and range of results of the three response variables obtained with the optimization Box-Behnken experimental designs for both reactions types in an evaluation of Diltiazem standard $5.544 \times 10^{-4} \mathrm{M}$, in methanol, at room temperature with a sodium hydroxide concentration of $12.500 \%$ for reaction type $\mathrm{A}$ and with a fixed volume of perchloric acid for reaction type B

Design variables

Reaction type

Reaction type A

Levels of design variables

Reaction type B

$6.250 \%$ and $18.750 \%$, center sample, $12.500 \%$ 0.250 and $0.500 \mathrm{~mL}$, center sample, $0.375 \mathrm{~mL}$

Volume of perchloric acid Concentration of sodium hydroxide

Concentration of ferric reagent $1.000 \%$ and $2.000 \%$, center sample, $1.500 \%$
$6.250 \%$ and $12.500 \%$, center sample, $9.375 \%$

$12.500 \%$ and $25.000 \%$, center sample, $18.750 \%$ $1.000 \%$ and $2.000 \%$, center sample, $1.500 \%$

Response variables

Reaction type

Reaction type A Reaction type B

Range of results obtained at maximum absorbance

\begin{tabular}{lll}
\hline Absorbance & $0.271-0.531$ a.u. & $0.451-0.555$ a.u. \\
Wavelength & $508-510 \mathrm{~nm}$ & $506-514 \mathrm{~nm}$ \\
Reaction time & $3-31 \mathrm{~s}$ & $3-29.5 \mathrm{~s}$
\end{tabular}

observed for reaction type $\mathrm{B}$. This result is due to the higher concentration of sodium hydroxide used in this reaction type.

Although, in this design the main effects cannot be distinguished from the two variable interactions, it is possible to observe that hydroxylamine, sodium hydroxide and ferric reagent concentrations provoke variation in the responses. When water is used as solvent of the standard Diltiazem solution a decrease in the response variables is observed and consequently methanol is the best solvent for standard and samples preparation. Because the reaction time is relatively small when the reaction is performed at room temperature and, taking into consideration that at room temperature the method becomes much more easy to be performed, this temperature was used subsequently. For the other variables the minimum volume of perchloric acid to neutralize the sodium hydroxide, the greater sodium hydroxide and ferric reagent concentrations should be used.

\subsubsection{Confirmatory screening experimental designs}

Taking into consideration the previous information, and in order to test the observations of the low resolution exploratory screening experimental designs, a $2^{4-1}$ confirmatory screening experimental designs were performed with nineteen experiments each-four continuous designs variables, two levels, two repetitions, three center samples and three response variables. With this design the two variable interactions are confounded and only the main effects can be rigorously estimated. For both reaction types the levels of the reaction temperature are maintained and only the variable solvent was fixed. Also, for both reaction types, due to the respective decrease of reaction time and wavelength associated with an absorbance increase, the higher levels of the variables sodium hydroxide and ferric reagent concentrations were lowest. For reaction type A both levels of the volume of perchloric acid are maintained and the low level of the hydroxylamine concentration was increased. For reaction type $B$ the levels of the hydroxylamine concentration were maintained. Table 2 shows the design variables studied, the levels of each one and the range of results obtained for the response variables.
Tables 3 and 4 show the analysis of the effects of the four factors on the three responses for the two reaction types. The analysis of these two tables show that the linear models used in the evaluation of the main and interaction effects are significant in the experimental domain for the responses absorbance and wavelength but not for the reaction time. Also, for the absorbance, all the main effects are statistically significant according to the HOIE test.

For the absorbance response, the analysis of the critical effects confirms the conclusions by the HOIE test. The hydroxylamine concentration, the volume of perchloric acid and sodium hydroxide concentration effects are statistical significant or possible statistical significant by the two tests used. Also, for this response variable and for the reaction type A, the ferric reagent concentration effect is statistical significant by the test based on an estimation of error by the interaction effects and possible statistical significant by the test based on the algorithm of Dong.

The results obtained with this fractional experimental design and presented in Tables 3 and 4 clarify the results present in Section 3.2.1. Consequently, these results support the use of a minimum volume of perchloric acid to neutralize the sodium hydroxide, room temperature, greater concentrations of sodium hydroxide and ferric reagent, as the more adequate conditions to obtain a greater sensibility in the response with a fast reaction time.

In order to obtain an optimum set of reagent concentrations, besides methanol as solvent also the room temperature was fixed, the response surfaces of the chemical reaction under investigation will be obtained using Box-Behnken and central composite designs.

\subsection{Optimization experimental designs}

\subsubsection{Box-Behnken design}

The Box-Behnken design has three variables, two levels, without repetitions, three center samples and three response variables (fifteen experiments each). Taking into consideration the information obtained in the previous screening designs the levels of the variables in this design were adjusted accord- 
Table 6 - Results obtained with the Box-Behnken experimental design for reaction type A, by analysis of (a) main effects and (b) response surfaces, through the response evaluation of a Diltiazem standard $5.544 \times 10^{-4} \mathrm{M}$, in methanol, with a sodium hydroxide concentration of $12.500 \%$ and at room temperature ${ }^{\mathrm{a}}$

(a) Main effects

\begin{tabular}{|c|c|c|c|c|c|c|c|c|c|}
\hline \multirow[t]{3}{*}{ Design variables } & \multicolumn{9}{|c|}{ Response variables } \\
\hline & \multicolumn{3}{|c|}{ Absorbance } & \multicolumn{3}{|c|}{ Wavelength } & \multicolumn{3}{|c|}{ Reaction time } \\
\hline & HOIE & Center & Coefficient $b$ & HOIE & Center & Coefficient $b$ & HOIE & Center & Coefficient $b$ \\
\hline Hydroxylamine & $28.286(0.001)$ & $128.704(0.008)$ & -0.071 & $0.000(1.000)$ & $\mathrm{np}(1.000)$ & 0.000 & $0.374(0.558)$ & $10.010(0.087)$ & -1.938 \\
\hline Perchloric acid & $14.435(0.005)$ & $65.681(0.015)$ & -0.051 & $1.500(0.256)$ & $\mathrm{np}(0.000)$ & +0.250 & $1.547(0.249)$ & $41.344(0.023)$ & +3.938 \\
\hline Ferric reagent & $11.330(0.010)$ & $51.552(0.019)$ & +0.045 & $1.500(0.256)$ & $\mathrm{np}(0.000)$ & -0.250 & $0.025(0.878)$ & $0.667(0.500)$ & -0.500 \\
\hline \multicolumn{10}{|c|}{ Global linear model (ANOVA) } \\
\hline F-ratio $(p)$ & $18.017(0.0006)$ & & & $1.000(0.441)$ & & & $0.649(0.606)$ & & \\
\hline Multiple correlation & 0.933 & & & 0.522 & & & 0.442 & & \\
\hline \multicolumn{10}{|c|}{ (b) Response surfaces } \\
\hline \multirow[t]{2}{*}{ Design variables } & & & \multicolumn{7}{|c|}{ Response variables } \\
\hline & & & Absorbance & & & Waveleng & & & Reaction time \\
\hline Estimate point & & & Maximum, 0.582 a.u. & & & Saddle, 510.12 & & & Saddle, $5.812 \mathrm{~s}$ \\
\hline Hydroxylamine (\%) & & & 9.433 & & & 12.500 & & & 13.809 \\
\hline Perchloric acid (mL) & & & 0.061 & & & 0.437 & & & 0.311 \\
\hline Ferric reagent (\%) & & & 2.067 & & & 1.250 & & & 1.471 \\
\hline \multicolumn{10}{|c|}{ Global quadratic model (ANOVA) } \\
\hline F-ratio $(p)$ & & & $42.374(0.000)$ & & & $1.156(0.413)$ & & & $1.085(0.445)$ \\
\hline Multiple correlation & & & 0.985 & & & 0.681 & & & 0.670 \\
\hline Quadratic effects [F-r & & & $17.550(0.0007)$ & & & $0.978(0.450)$ & & & $1.252(0.354)$ \\
\hline Lack of fit [F-ratio (p) & & & $1.293(0.497)$ & & & $\mathrm{np}(0.000)$ & & & $24.878(0.039)$ \\
\hline
\end{tabular}

a In HOIE and "center" tests the F-ratio is presented for each main effect and interaction; Coefficient $b$ : $b$ regression coefficient of each effect or interaction in the model; np: not possible the calculus; in brackets is presented the probability value $(p)$, for a $5 \%$ significance level, the number in brackets indicates in the main or interactions effects evaluation the probability of a main or interactions effects be significant by HOIE or "center" tests or in the global linear and quadratic model evaluation the probability of the model be significantly valid. 
Table 7 - Results obtained with the Box-Behnken experimental design for reaction type B, by analysis of (a) main effects and (b) response surfaces, through the

response evaluation of a Diltiazem standard $5.544 \times 10^{-4} \mathrm{M}$, in methanol, with a fixed volume of perchloric acid and at room temperature ${ }^{a}$

(a) Main effects

\begin{tabular}{|c|c|c|c|c|c|c|c|c|c|}
\hline \multirow[t]{3}{*}{ Design variables } & \multicolumn{9}{|c|}{ Response variables } \\
\hline & \multicolumn{3}{|c|}{ Absorbance } & \multicolumn{3}{|c|}{ Wavelength } & \multicolumn{3}{|c|}{ Reaction time } \\
\hline & HOIE & Center & Coefficient $b$ & HOIE & Center & Coefficient $b$ & HOIE & Center & Coefficient $b$ \\
\hline Hydroxylamine & $0.005(0.945)$ & $0.024(0.892)$ & -0.0008 & $1.500(0.256)$ & $0.375(0.603)$ & -0.500 & $0.831(0.389)$ & $0.604(0.519)$ & -2.937 \\
\hline Sodium hydroxide & $0.000(0.988)$ & $0.001(0.976)$ & -0.0002 & $1.500(0.256)$ & $0.375(0.603)$ & +0.500 & $0.046(0.836)$ & $0.033(0.873)$ & +0.688 \\
\hline Ferric reagent & $4.547(0.066)$ & $21.152(0.044)$ & +0.024 & $1.500(1.000)$ & $0.000(1.000)$ & 0.000 & $0.002(0.970)$ & $0.001(0.977)$ & -0.125 \\
\hline \multicolumn{10}{|c|}{ Global linear model (ANOVA) } \\
\hline F-ratio $(p)$ & $1.518(0.282)$ & $1.000(0.441)$ & $0.293(0.830)$ & & & & & & \\
\hline Multiple correlation & 0.602 & 0.522 & 0.315 & & & & & & \\
\hline
\end{tabular}

\begin{tabular}{|c|c|c|c|}
\hline \multirow[t]{2}{*}{ Design variables } & \multicolumn{3}{|c|}{ Response variables } \\
\hline & Absorbance & Wavelength & Reaction time \\
\hline Estimate point & Saddle, 0.493 a.u. & Maximum, $511.440 \mathrm{~nm}$ & Saddle, $17.448 \mathrm{~s}$ \\
\hline Hydroxylamine (\%) & 9.244 & 8.705 & 8.733 \\
\hline Sodium hydroxide (\%) & 18.714 & 20.089 & 18.109 \\
\hline Ferric reagent (\%) & -0.026 & 1.500 & 1.385 \\
\hline \multicolumn{4}{|c|}{ Global quadratic model (ANOVA) } \\
\hline F-ratio $(p)$ & $1.009(0.481)$ & $0.652(0.510)$ & $0.637(0.700)$ \\
\hline Multiple correlation & 0.656 & 0.645 & 0.569 \\
\hline Quadratic effects [F-ratio $(p)]$ & $0.432(0.736)$ & $1.333(0.330)$ & $0.982(0.448)$ \\
\hline Lack of fit [F-ratio $(p)]$ & $5.601(0.159)$ & $0.250(0.921)$ & $0.637(0.717)$ \\
\hline
\end{tabular}

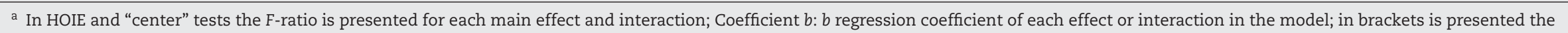
probability value (p), for a $5 \%$ significance level, the number in brackets indicates in the main or interactions effects evaluation the probability of a main or interactions effects be significant by HOIE or "center" tests or in the global linear and quadratic model evaluation the probability of the model be significantly valid. 
Table 8 - Levels of design variables used and range of results of the three response variables obtained with the optimization central composite design in an evaluation of Diltiazem standard $5.544 \times 10^{-4} \mathrm{M}$, in methanol, with sodium hydroxide concentration of $18.750 \%$, with a fixed volume of perchloric acid and at room temperature

Design variables

Levels of design variables

Concentration of hydroxylamine

Concentration of ferric reagent

Response variables
Cube samples: $6.250 \%$ and $12.500 \%$, star samples: $4.956 \%$ and $13.794 \%$, center samples: $9.375 \%$ Cube samples: $1.500 \%$ and $2.500 \%$, star samples: $1.293 \%$ and $2.707 \%$, center samples: $2.000 \%$

Range of results obtained at maximum absorbance ing to the following: the high level of the volume of perchloric acid was decreased; both levels of the concentration of sodium hydroxide and ferric reagent were increased; the low level of the concentration of hydroxylamine was increased. Table 5 presents the design variables, the corresponding levels studied and the range of results obtained at maximum absorbance for the response variables under a Box-Behnken design for the two reaction types.

The results obtained with these optimization designs are presented in Tables 6 and 7. The main effects of the design variables in the response variables confirm the previous results. For the reaction type A, the linear model used for the evaluation of the main and interactions effects for the response absorbance is significant in the experimental domain and all the main effects for this response variable are statistically significant according to the HOIE test.

With the two reaction types from the analysis of the three absorbance response surfaces a maximum of the response absorbance could be defined. For the reaction type $\mathrm{A}$, is obtained in all the response surfaces, a maximum at a hydroxylamine concentration of $9.375 \%$, a perchloric acid volume of $0.250 \mathrm{~mL}$ and a ferric reagent concentration of $2.000 \%$. For the reaction type $\mathrm{B}$ a maximum point at a hydroxylamine concentration of $9.115 \%$, a sodium hydroxide concentration of $18.750 \%$ and a ferric reagent concentration of $2.000 \%$. These results indicate that $9.375 \%$ is the optimum hydroxylamine concentration for a sodium hydroxide concentration of $18.750 \%$ with a minimum volume of perchloric acid to neutralize the sodium hydroxide and eventually a greater concentration of ferric reagent.

\subsubsection{Central composite design}

A last optimization design was done to obtain response surfaces for the concentration of hydroxylamine and ferric reagent. A central composite design with two variables, four levels, without repetitions, five center samples and three response variables was used (thirteen experiments). Relatively to the levels evaluated in the Box-Behnken design the levels of hydroxylamine concentration are the same and the low and high levels of ferric reagent concentration were increased. The other experimental variables were fixed at the optimum values previously defined: solution of Diltiazem prepared in methanol; room temperature; $18.750 \%$ sodium hydroxide concentration; minimum volume of perchloric acid to neutralize the sodium hydroxide. Table 8 presents the design variables and corresponding levels under study.

The results obtained with this optimization design are presented in Table 9. The analysis of Table 9 shows that the linear model for the absorbance response, is statistically significant. Also, the variable that affects markedly the absorbance is the ferric reagent concentration.

Fig. 2 shows the response surfaces for the three response variables obtained with the central composite design. The analysis of this figure shows that, with the exception of the reaction time, an optimum is clearly defined in the response surface. Response surface from Fig. 2a confirms an optimum of hydroxylamine concentration of $9.375 \%$. From the analysis of the three response surfaces the following concentration ranges of ferric reagent are detected: $2.000-2.500 \%$ for maximum absorbance at a higher wavelength; and, 1.500-2.000\% for a minimum reaction time. A value for the ferric reagent concentration of $2.000 \%$ seems to be the more adequate for a Diltiazem determination with greater sensibility and minimum reaction time.

\subsection{Final evaluation}

A final univariate evaluation was done with the increase of the ferric reagent concentration from $0.500 \%$ to $5.000 \%$. The results found are presented in Fig. 3. An increase in the absorbance until a ferric reagent concentration of $2.000 \%$ followed by absorbance stabilization up to a ferric reagent concentration of $3.000 \%$ and posterior decrease was observed.

The optimized reaction conditions (small reaction time with higher absorbance and wavelength) for the Diltiazem determination are: methanol as solvent; room temperature; hydroxylamine concentration $=9.375 \%$; sodium hydroxide concentration $=18.750 \%$; ferric reagent concentration $=2.000 \%$; volume of perchloric acid minimum to neutralize the sodium hydroxide.

With these optimized conditions, with five determinations of a Diltiazem standard $5.544 \times 10^{-4} \mathrm{M}$ (R.S.D. $=0.847 \%$ ) an average of absorbance 0.700 a.u., wavelength $512 \mathrm{~nm}$ and a reaction time of $10.5 \mathrm{~s}$ were found.

Using a series of Diltiazem standards $\left(5.677 \times 10^{-5}\right.$, $1.100 \times 10^{-4}, \quad 2.235 \times 10^{-4}, \quad 4.435 \times 10^{-4}, \quad 6.670 \times 10^{-4}$ $8.870 \times 10^{-4}$ and $1.110 \times 10^{-3} \mathrm{M}$ ) the following figures of merit of the calibration curve were obtained: linear calibration func- 
Table 9 - Results obtained with the central composite experimental design, by analysis of (a) main effects and (b) response surfaces, through the response evaluation of a Diltiazem standard $5.544 \times 10^{-4} \mathrm{M}$, in methanol, with sodium hydroxide concentration of $18.750 \%$, with a fixed volume of perchloric acid and at room temperature ${ }^{\mathrm{a}}$

\begin{tabular}{|c|c|c|c|c|c|c|c|c|c|}
\hline \multirow[t]{3}{*}{ Design variables } & \multicolumn{9}{|c|}{ Response variables } \\
\hline & \multicolumn{3}{|c|}{ Absorbance } & \multicolumn{3}{|c|}{ Wavelength } & \multicolumn{3}{|c|}{ Reaction time } \\
\hline & HOIE & Center & Coefficient $b$ & HOIE & Center & Coefficient $b$ & HOIE & Center & Coefficient $b$ \\
\hline Hydroxylamine & $0.167(0.753)$ & $0.001(0.974)$ & -0.001 & $1.000(0.500)$ & $1.250(0.326)$ & +0.500 & $0.524(0.601)$ & $1.018(0.370)$ & -2.625 \\
\hline Ferric reagent & $499.354(0.029)$ & $3.497(0.135)$ & +0.027 & $9.000(0.205)$ & $11.250(0.029)$ & +1.500 & $0.344(0.663)$ & $0.667(0.460)$ & -2.125 \\
\hline \multicolumn{10}{|c|}{ Global linear model (ANOVA) } \\
\hline F-ratio $(p)$ & $249.760(0.045)$ & & & $5.000(0.302)$ & & & $0.434(0.732)$ & & \\
\hline Multiple correlation & 0.999 & & & 0.953 & & & 0.682 & & \\
\hline \multicolumn{10}{|c|}{ (b) Response surfaces } \\
\hline \multirow[t]{2}{*}{ Design variables } & & & \multicolumn{7}{|c|}{ Response variables } \\
\hline & & & Absorbance & & & Wavelength & & & Reaction time \\
\hline Estimate point & & & Maximum, 0.586 a.u. & & & Maximum, 512.71 & & & Saddle, $12.686 \mathrm{~s}$ \\
\hline Hydroxylamine (\%) & & & 9.060 & & & 11.398 & & & 8.252 \\
\hline Ferric reagent (\%) & & & 2.550 & & & 2.111 & & & 2.854 \\
\hline \multicolumn{10}{|c|}{ Global quadratic model (ANOVA) } \\
\hline F-ratio $(p)$ & & & $0.959(0.500)$ & & & $0.888(0.536)$ & & & $1.552(0.288)$ \\
\hline Multiple correlation & & & 0.638 & & & 0.623 & & & 0.725 \\
\hline Quadratic effects [F- & & & $0.948(0.432)$ & & & $1.112(0.381)$ & & & $0.396(0.687)$ \\
\hline Lack of fit $[F$-ratio $(p)$ & & & $1.500(0.343)$ & & & $9.649(0.027)$ & & & $1.732(0.298)$ \\
\hline
\end{tabular}


(a) Absorbance

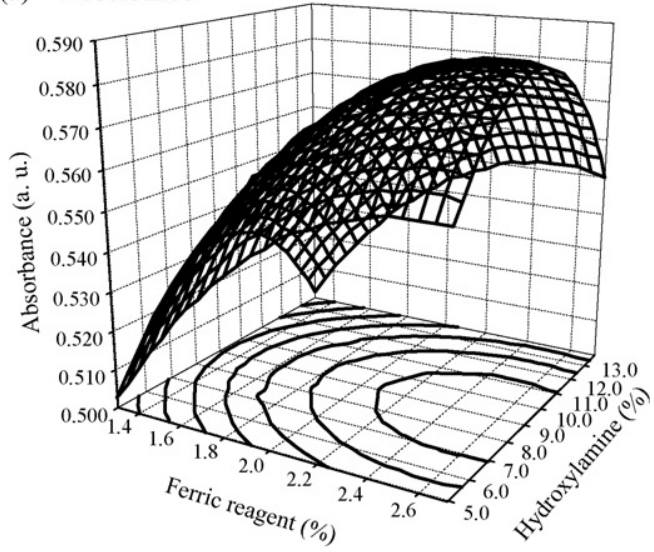

(b) Wavelength

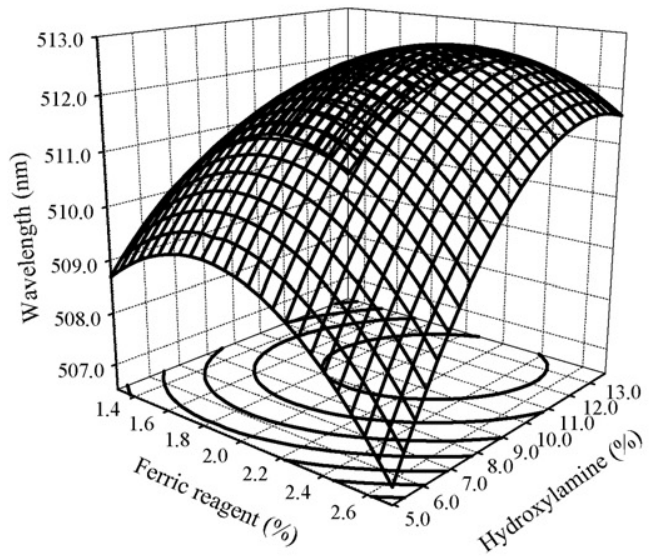

(c) Reaction time

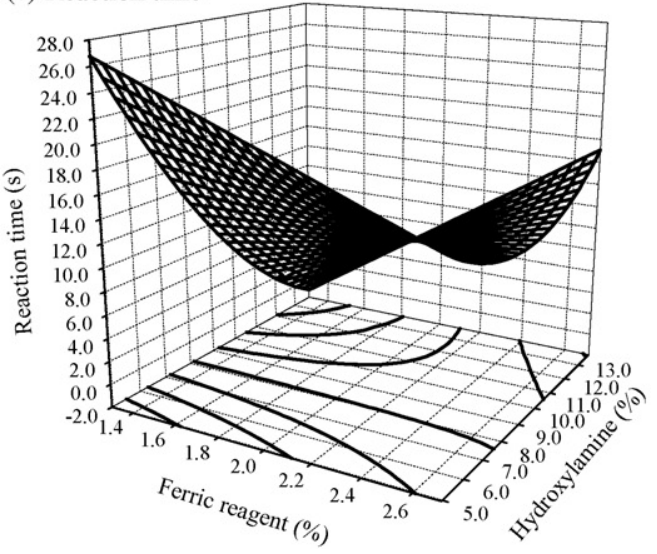

Fig. 2 - Response surfaces of (a) absorbance, (b) wavelength and (c) reaction time obtained with the central composite experimental design, through the response evaluation of a Diltiazem standard $5.544 \times 10^{-4} \mathrm{M}$, in methanol, with sodium hydroxide concentration of $18.750 \%$, with a fixed volume of perchloric acid and at room temperature.

tion, $y$ (a.u. $)=956.472 x(M)+0.022(m=7)$; linear work range, $5.677 \times 10^{-5} \mathrm{M}$ to $1.110 \times 10^{-3} \mathrm{M}(25-500 \mathrm{mg} / \mathrm{L})$; residual standard deviation $=0.023$; linear correlation coefficient $=0.9986$; detection limit $\left(3 \mathrm{~s}_{\mathrm{a}} / \mathrm{b}\right)=4.463 \times 10^{-5} \mathrm{M}$.

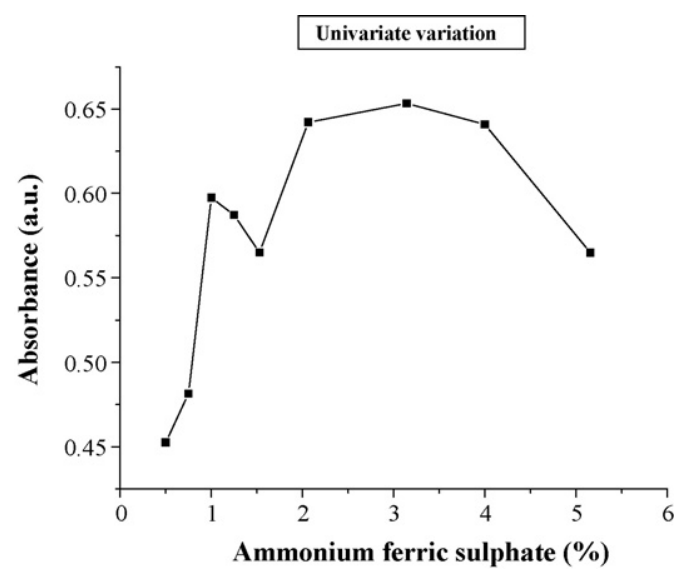

Fig. 3 - Univariate variation of absorbance in function of ferric reagent concentration.

\section{Conclusions}

The main effects that affect the response variables are the concentration of hydroxylamine, sodium hydroxide and of ammonium ferric sulphate. In all the experimental designs evaluated they affect essentially the absorbance. The temperature of the reaction also affects the response variables but, because the method becomes much easier to perform at room temperature, this temperature was used subsequently. For a level of $5 \%$, the interactions between the design variables at the levels evaluated are significant for screening the experimental designs but not significant for optimization the experimental design evaluated.

The optimal conditions observed for this reaction were: (i) standard solution in methanol, (ii) hydroxylamine $9.375 \%$, (iii) sodium hydroxide $18.750 \%$, (iv) ammonium ferric sulphate $2.000 \%$, (v) minimum volume of perchloric acid $7 \%(\mathrm{w} / \mathrm{w})$ to neutralize the sodium hydroxide and (vi) room temperature. These optimal reaction conditions allow a low reaction time at maximum sensitivity with a linear response of the absorbance as function of the concentration.

\section{REFERENCES}

[1] A.I. Vogel, in: S.A. Livro Técnico (Ed.), Química orgânica-Análise orgânica qualitative, Indústria e comércio, Rio de Janeiro, 1984, pp. 1118-1120.

[2] R.F. Goddu, N.F. LeBlanc, C.M. Wright, Anal. Chem. 27 (1955) 1251.

[3] W.P. Jencks, J. Am. Chem. Soc. 80 (1958) 4585.

[4] R.E. Notari, J.W. Munson, J. Pharm. Sci. 58 (1969) 1060.

[5] United States Pharmacopeial Convention, USP 29 (Ed.), NF 24-The United States Pharmacopeia, The national Formulary, Rockville, 2006.

[6] European Pharmacopeia, Council of Europe ed. 1st volume and supplement 5.1, 2004.

[7] British Pharmacopeia, HMSO Her Majesty's Stationary Office ed., 2005.

[8] D.J. Mazzo, C.L. Obetz, J. Shuster, Analytical Profiles of Drug Substances and Excepients, vol. 23, Academic Press, New York, 1994, pp. 53-98.

[9] D. Zivanov-Stakic, D. Agbaba, L.J. Ciric, Il Farm. 47 (1993) 393. 
[10] B.V. Kamath, K. Shivram, A.C. Shah, J. Pharm. Biomed. Anal. 11 (1993) 407.

[11] M. Ayad, A. Shalaby, H. Abdellatef, M. Hosny, Anal. Bioanal. Chem. 376 (2003) 710.

[12] C. Sastry, K. Sreedhar, M. Reddy, D. Sankar, Indian J. Pharm. Sci. 57 (1995) 170.

[13] N. Rahman, S. Hejaz-Azmi, J. Pharm. Biomed. Anal. 24 (2000) 33.

[14] K. Sreedhar, C. Sastry, M. Reddy, D. Sankar, Indian Drugs 32 (1995) 90.

[15] Y. Agrawal, K. Shivramachandra, B. Rao, Indian J. Pharm. Sci. 54 (1992) 218.

[16] B. Kamath, K. Shivram, The East. Pharm. 35 (1992) 121.

[17] B. Kamath, K. Shivram, Indian Drugs 29 (1991) 50.

[18] R. Pietras, D. Kowalczuk, H. Hopkala, Acta Pol. Pharm. 61 (2004) 315.

[19] T. Lundstedt, E. Seifert, L. Abramo, B. Thelin, Å. Nyström, J. Pettersen, R. Bergman, Chem. Intell. Lab. Syst. 42 (1998) 3.

[20] J. Berridge, Analyst 112 (1987) 385.
[21] J.C.G. Esteves da Silva, J.R.M. Dias, J.M.C.S. Magalhães, Anal. Chim. Acta 450 (2001) 175.

[22] P.B.M. Pinheiro, J.C.G. Esteves da Silva, Anal. Bioanal. Chem. $382(2005) 341$.

[23] P.M.S.M. Rodrigues, J.C.G. Esteves da Silva, M.C.G. Antunes, Anal. Chim. Acta 595 (2007) 266.

[24] J.M.M. Leitão e, J.C.G. Esteves da Silva, Chem. Intell. Lab. Syst. 89 (2007) 90.

[25] P. Araujo, R.G. Brereton, Trends Anal. Chem. 15 (1996) 26.

[26] D.L. Massart, B.G.M. Vandeginste, L.M.C. Buydens, S.D. Jong, P.G. Lewi, J. Smeyers-Verbeke, Handnook of Chemometrics and Qualimetrics: Part A (Data Handling in Science and Technology, vol. 20 A), Elsevier, Amsterdam, 1997, pp. 643-738.

[27] P. Araujo, R.G. Brereton, Trends Anal. Chem. 15 (1996) 63.

[28] Y.V. Heyden, A. Nijhuis, J. Smeyers-Verbeke, B.G.M. Vandeginste, D.L. Massart, J. Pharm. Biomed. Anal. 24 (2001) 723.

[29] F. Dong, Stat. Sinica 3 (1993) 209. 